\title{
THE RECONSTRUCTION OF THE OBJECT SURFACE USING CONFOCAL MICROSCOPE WITH HYPERCHROMATIC LENS
}

\author{
Pavel Starha, Jana Prochazkova, Dalibor Martisek
}

\author{
Brno University of Technology \\ Institute of Materials Science and Engineering, NETME centre \\ Technicka 2, Brno \\ Czech Republic \\ starha@fme.vutbr.cz, prochazkova.j@fme.vutbr.cz, martisek@fme.vutbr.cz
}

\begin{abstract}
The usage of a tandem-scanning reflected-light microscope is not common but this technology offers the wide possibilities in the area of the surface reconstruction. This article presents a method that can reconstruct the $3 D$ relief from a single image with a known calibrated set of images. The method uses the property that the points in the same height are visualized in the same color and we propose the nonlinear optimization to find this dependence. Subsequently, the low brightness parts are added by circular partial convolution.
\end{abstract}

Keywords: confocal microscope, surface reconstruction, least square method, partial convolution, Gauss filter, minimization, hyperchromatic lens.

\section{Introduction}

Three-dimensional reconstructions of object surfaces play an important role in many branches and a confocal microscope is a standard tool for 3D reconstruction of the microscopic objects. There is a wide area of the applications in the natural sciences because the confocal microscope essentially removes the out-of-focus light by inserting a pinhole at the image plane such that out-of-focus light does not reach the detector. Only light focused on the pinhole passes through it, all other light is scattered. Due to this property, modern confocal systems are able to work simply, effectively and precisely in e.g. biomedical research [8]. Also in the engineering, there is a need of morphological analysis of fracture surfaces that reveals information on mechanical properties of construction materials (see $[1,2,3,4]$ ) or with the roughness of machined metal surfaces [5, 6].

The article presents an interesting method of 3D reconstruction using the confocal microscope with hyperchromatic lens. This Tandem-scanning reflected-light microscope was found by M. Petran and M. Hadravsky in 1964. The scanning technology is clearly described in [7]. Both the object plane and the image plane are scanned in tandem so that only light reflected from the object plane is included in the image. The object is illuminated with nearly incoherent light passing through holes in one side of a rotating scanning disk (Nipkow wheel) which is imaged by the objective into the object plane. Reflected-light images of these spots are conducted to the opposite side of the same disk. The hyperchromatic lens cause that different depths of field (DOF) are visible with different colors - hue. Therefore, the analysis of the hue and height dependence plays the crucial role of the $3 \mathrm{D}$ reconstruction. Also, we use the non-linear optimization and circular partial convolution to the accuracy improvement.

\section{Problem Formulation}

Built-in chromatic imperfection of the confocal microscope lens is the base of the proposed method. The color (hue) of the reflected light is determined by the different distance of the lens and surface (we call it reference distance). So that, we first compute this non-linear dependence of the hue and the reference distance of the image high. Subsequently, we apply the result function to compute the correct 3D reconstruction of the surface from a single image. The problem causes the pixels with low brightness. The high profile of these missing pixels is computed with partial convolution with the Gaussian circular core.

\subsection{The hue and the reference distance dependence}

The set of calibrated images is used for the computation of the hue and the reference distance dependence. Images of mirror reflections fulfill the requirements about local constant surface height so that we use them as the suitable calibrated image set $\mathcal{I}_{k}, k=1, \ldots, n$. The size of all images is $1340 \times 976$ and we acquire them with the step $0.005 \mathrm{~mm}$. The step size corresponds to the reference distance change between two subsequent images. 
Figure Fig. 1 shows forty different images of the mirror. Afterward, we calibrate all images by dark-frame subtraction.

The determination of the hue and reference distance dependence consists of three steps:

1. the choice of reference image $\mathcal{I}_{\text {ref }}$,

2. the identification of same hue $H_{\text {ref }}$ pixels $p_{i j}^{r e f}, i, j \in \mathcal{N}$ in the reference image $\mathcal{I}_{\text {ref }}$, we suppose that the hue in the image is homogeneous.

3. the identification of the hue $H_{k}$ of pixels $p_{i j}^{k}$ in same positions as $p_{i j}^{r e f}$ in remaining images in set $\mathcal{I}_{k}$.

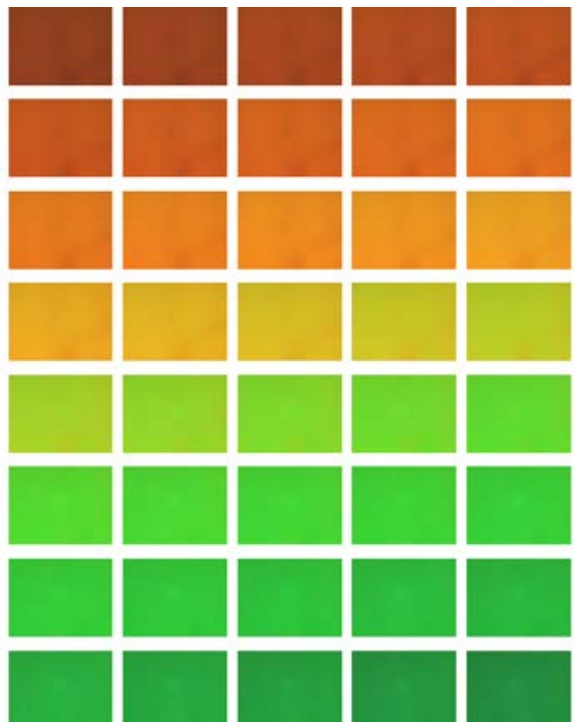

Figure 1: The calibrated images of the mirror.

The values of $H_{k}$ and the number of image $x, x=1, \ldots, n$ (reference distance) give the required dependence. Due to the graph shape on Fig. 2, we choose a non-linear minimization function based on the trigonometric function arctan.

$$
H(x)=C\left({\frac{\arctan (A x+B)^{E}}{\pi}}^{E}\right)+D
$$

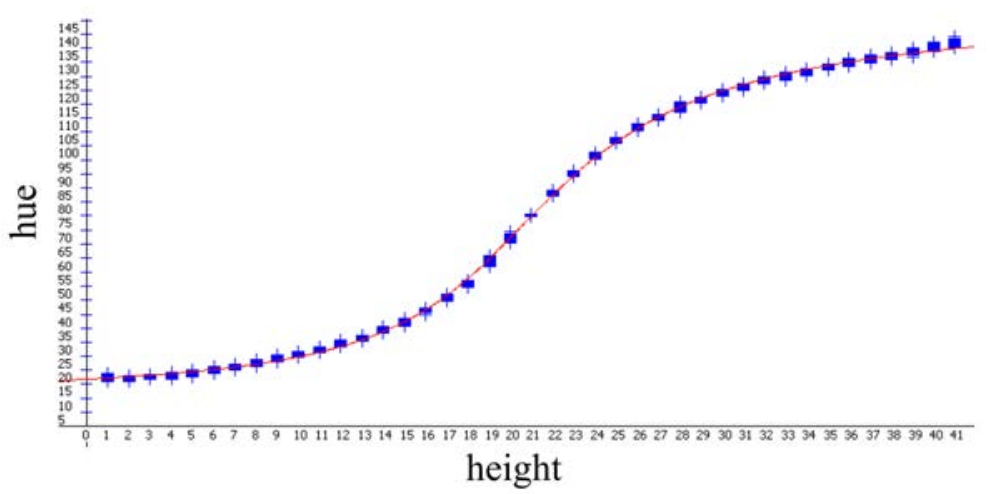

Figure 2: The dependence of the hue and the relative reference distance of the image

Note that the hue value of one tested calibrated image is the average of all pixels hue values. The computation of the unknown values $A, B, C, D, E$ is based on the gradient method and Newton method. The dependence of the reference height of the image $x$ and the hue $H$ is done by inversion of Eq. (1):

$$
x=\frac{\tan \left(\pi\left(\frac{H-D}{C}-\frac{\pi}{2}\right)^{\frac{1}{E}}-B\right)}{A}
$$




\subsection{Convolution and Gaussian Core}

In Sect. 2.1 we identify the dependence of the relative reference height and the hue. Now, it is possible to use this function to reconstruct the surface given by single image $\mathcal{I}$. The problem causes the evaluation of pixels with the low brightness intensity. They contain the high color error that is caused by additive noise. These pixels are on the distinct elevation change because the reflected light in these parts passed the lens. It is not possible to compute their correct hue $H$ using Eq. (2). So that we chose the partial convolution with the adaptive Gaussian kernel as a suitable tool for the computation of these false pixels. The following definitions describe necessary mathematical background [9].

Definition. Let $f(x, y), g(x, y) \in \mathcal{L}\left(\mathrm{R}^{2}\right)$. The function $h(x, y)$ defined as:

$$
h(x, y)=f(x, y) * g(x, y)=\iint_{\mathrm{R} \times \mathrm{R}} f(u, v) g(x-u, y-v) d u d v
$$

is called convolution of the function $f(x, y)$ with convolution kernel $g(x, y)$.

Definition. Let $f(x, y), g(x, y) \in \mathcal{L}\left(\mathrm{R}^{2}\right)$ and $x, y \in \mathrm{Z}$. The function $h(x, y)$ :

$$
h(x, y)=f(x, y) * g(x, y)=\sum_{k} \sum_{l} f(k, l) g(x-k, y-l)
$$

is called discrete convolution.

Note that the Eq. (4) can be rewritten due to commutative property of convolution as

$$
h(x, y)=f(x, y) * g(x, y)=\sum_{k} \sum_{l} f(x-k, y-l) g(k, l)
$$

The notation of Eq.(5) is commonly used in programming. In the case of missing parts of definition domain, it is necessary to apply the partial convolution.

Definition. Let $f(x, y), g(x, y) \in \mathcal{L}\left(\mathrm{R}^{2}\right)$ and $D(f) \subset \mathrm{R}^{2}, D(g) \subset \mathrm{R}^{2}$. The partial convolution $h(x, y)$ with convolution kernel $g(x, y)$ is defined as:

$$
h(x, y)=f(x, y) * g(x, y)=\frac{\iint_{D(f)} f(u, v) g(x-u, y-v) d u d v}{\iint_{D(f)} g(x-u, y-v) d u d v}
$$

Definition. Two-variable Gauss function is defined as

$$
f(x, y)=a \exp \left\{-\left(\frac{\left(x^{2}-\mu_{x}\right)}{2 \sigma_{x}^{2}}+\frac{\left(y^{2}-\mu_{y}\right)}{2 \sigma_{y}^{2}}\right)\right\}
$$

where $a, \sigma_{x}, \sigma_{y}>0$ and $\mu_{x}, \mu_{y} \in \mathrm{R}$.

We set $\mu_{x}=\mu_{y}=0, \sigma_{x}=\sigma_{y}=\sigma$ and the value $a$ is chosen to normalize the kernel matrix, i.e. sum of all kernel matrix values is equal to one. The value $a$ is written as:

$$
a=\frac{1}{\sum_{k=-m}^{m} \sum_{l=-n}^{n} g[k+m, l+n]} .
$$

and Gauss function from Eq.(7) is of the form:

$$
f(x, y)=a \exp \left\{-\frac{x^{2}+y^{2}}{2 \sigma^{2}}\right\}, \text { where } a, \sigma>0 .
$$

Commonly, the square, rectangle, circle or ellipse are used as the shape of the convolution kernel. The selected shape depends on the solved task and a circle fulfills our requirements well. Let $r>0$ be the radius of the circle and we compute the circular Gaussian convolution with convolution matrix $G\left(\sigma=1, \mu_{x}=\mu_{y}=0\right.$, size $5 \times 5)$ :

$$
G_{L}=\frac{1}{5.4388}\left(\begin{array}{ccccc}
0 & 0 & 0.1353 & 0 & 0 \\
0 & 0.3679 & 0.6065 & 0.3679 & 0 \\
0.1353 & 0.6065 & 1 & 0.6065 & 0.1353 \\
0 & 0.3679 & 0.6065 & 0.3679 & 0 \\
0 & 0 & 0.1353 & 0 & 0
\end{array}\right)
$$




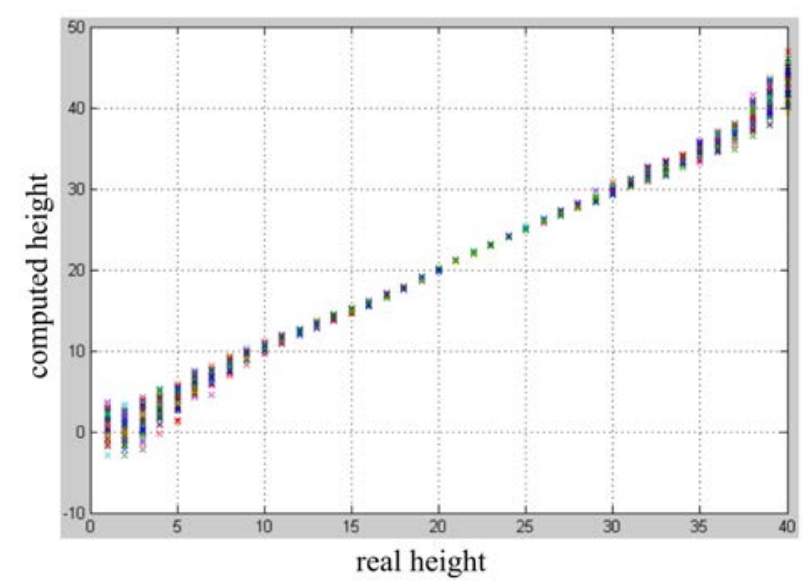

Figure 3: The analysis of the real and computed height.
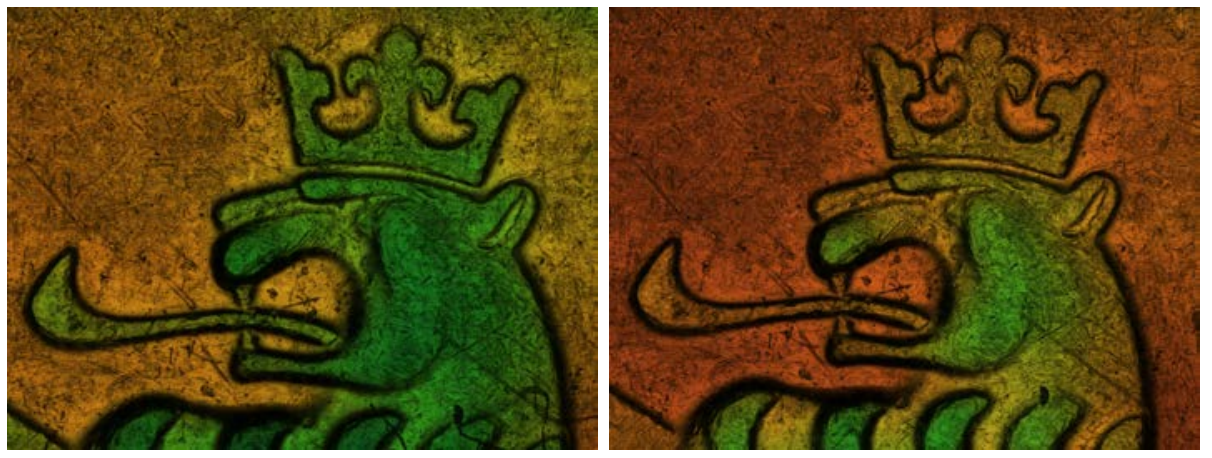

Figure 4: Two testing images of the coin.

\section{Problem Solution}

The calibration set of the mirror reflections $\mathcal{I}_{k}$ contains 40 images $(k=1, \cdots 40)$ and the measuring scale is 1 pixel $=0.0018408425 \mathrm{~mm}$. The dependence of the reference distance $x$ on the hue $H$ is computed by Eq. (2) using the image set $\mathcal{I}_{k}$. Fig. 3 shows the dependence of the real and computed altitude and it is clearly visible that the best reference distance is in the interval $\langle 10,32\rangle$, i.e. $H \in\langle 30,125\rangle$.

We use a part of the Czech one crown coin (lion head) as the testing object. Two testing 16-bit images are in Fig. 4. We set the minimal hue value $H_{\text {min }}$. The pixels with the hue that is lower than $H_{\text {min }}$ are skipped. As mentioned before, these pixels contain a large color error due to their position in the scanning object. They are on the steep parts of the relief so that the reflected light is out of the lens.

We process the images using Eq.(2) (dependence of the hue and the reference distance) and we reconstruct the values of the pixel height for non-excluded pixels. The computed values in Eq. (2) are:

$$
A=0.493951 B=-0.023575 C=4.714602 \quad D=0.335256 \quad E=1.293282
$$

The histogram describes the absolute frequency of particular colors in the reconstructed image. Fig. 5 shows the high profiles of two testing images and corresponding histograms. The histogram of the first image (Fig. 5, left) is optimal so that the first image is in the ideal position. The second image (the histogram in Fig. 5, right) has the shifted interval of hue $H$.

Subsequently, the problematic pixels with low brightness are evaluated using the partial convolution with the Gaussian circular kernel. The theory is described in Sec. 2.2. The value $\sigma$ in Eq. (7) is responsible for the interpolation properties and to the surface relief smoothing as well. It is necessary to use this convolution more times; firstly, with the small kernel and small value of $\sigma$ to dispose the unknown parts of the image. Lately, decrease the values of the $\sigma$ and size of the kernel to visualize the edge parts. The height profiles of these convolutions are in Fig. 6, 7 .

The computed reliefs of the coin reconstructed from images in Fig. 4 are displayed using library OpenGL and the result surfaces are in Fig. 8 . 


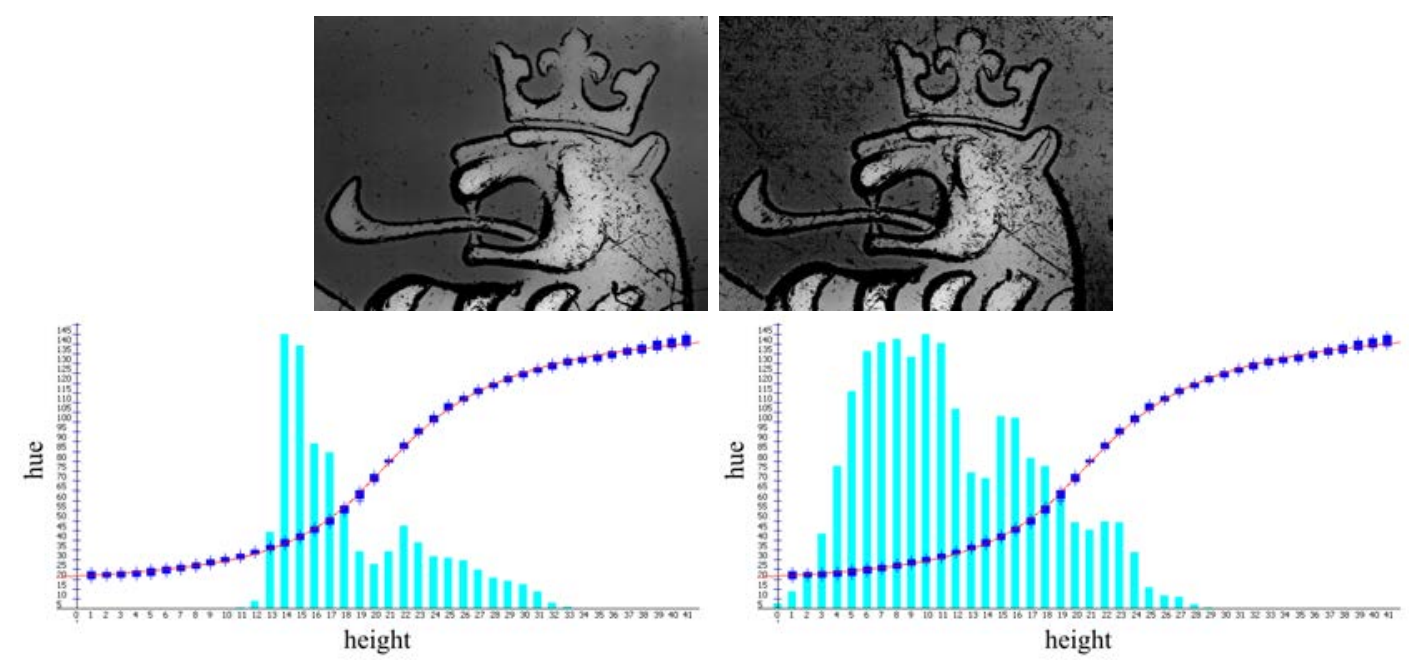

Figure 5: The height profile of two testing images and corresponding histograms.
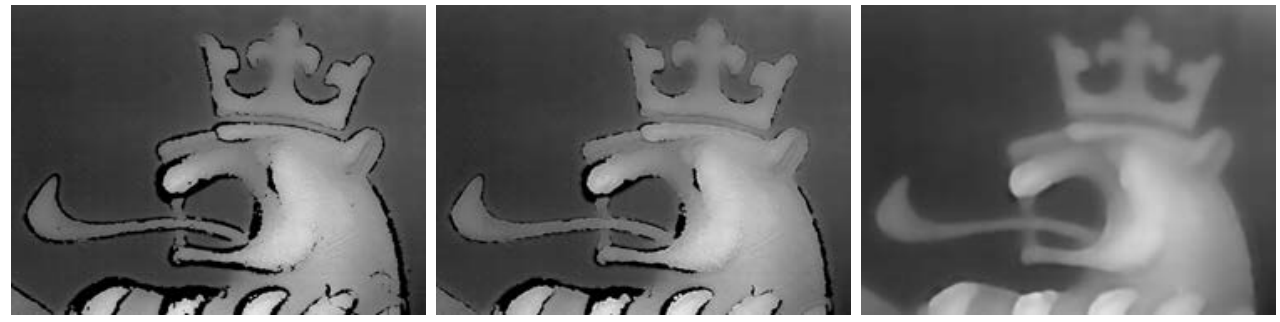

Figure 6: Different value of $\sigma$ and its influence on the shape of the result high image.
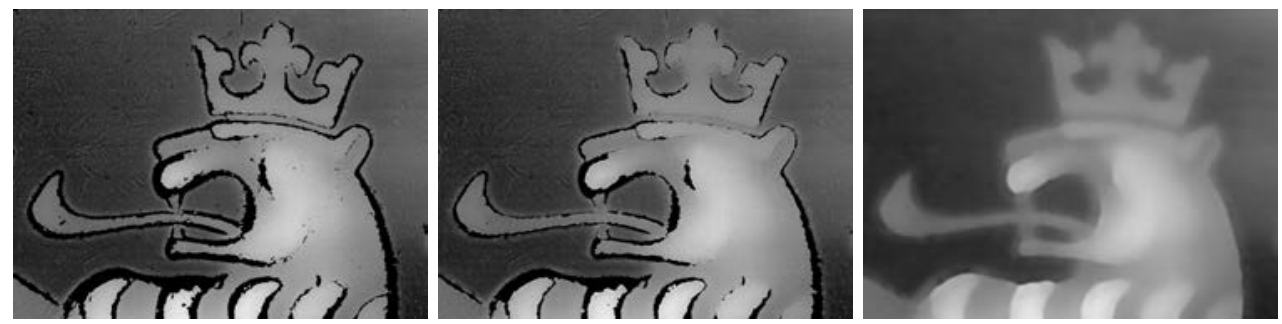

Figure 7: Different value of $\sigma$ and its influence on the shape of the result high image.
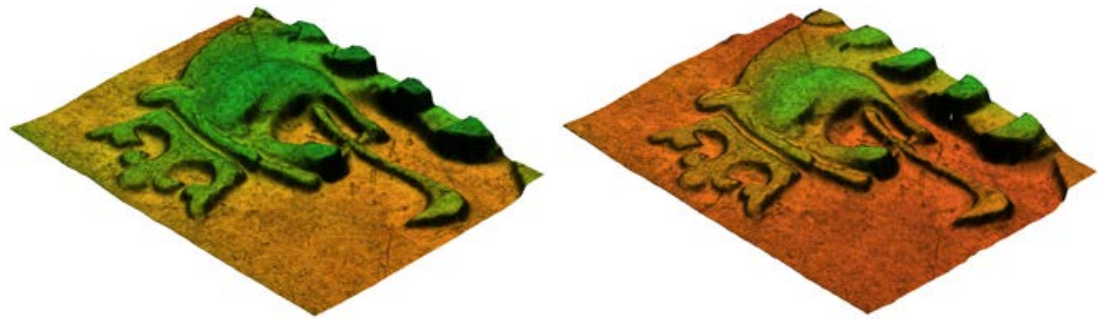

Figure 8: The computed 3D surface from single image using OpenGL.

\section{Conclusion}

The relief reconstructions from images are very interesting part of the image processing. We present the key description of the method that is able to reconstruct the 3D surface from the single image. The essential part is the set of the calibrated images that gives the dependence of the hue and the image altitude. With the computed dependence we can find the pixels altitude in most of the pixels. We suppose that the hue in the reference images is homogeneous but the real hue values have non-homogeneous range. The non-homogeneous dependence will give more accurate results. The places with a large difference in the profile are problematic. We describe the solution to reconstruct these pixels using the partial convolution with circular Gauss kernel. The proposed methods were tested on the images of the coin with lion relief and the results provide the precise $3 \mathrm{D}$ relief. 
Acknowledgement: The authors acknowledge support from Project LO1202 by financial means from the Ministry of Education, Youth and Sports, the National Sustainability Programme I.

\section{References}

[1] Lange,D.A., Jennings,H.M., Shah,S.P.: Analysis of surface-roughness using confocal microscopy. J. Mater. Sci. 28(14), pp. 3879-3884 (1993). DOI 10.1007/BF00353195

[2] Lange,D.A., Jennings,H.M., Shah,S.P.: Relationship between fracture surface roughness and fracture behavior of cement paste and mortar. J. Am. Ceram. Soc. 76(3), pp. 589-597 (1993).

[3] Zampini,D., Jennings,H.M., Shah, S.P.: Characterization of the paste-aggregate interfacial transition zone surface-roughness and its relationship to the fracture-toughness of concrete. J. Mater. Sci. 30(12), pp. 31493154 (1995).

[4] Lange, D.A., Quyang,C., Shah, S.P.: Behavior of cement-based matrices reinforced by randomly dispersed microfibers. Adv. Cem. Based Mater. 3(1), 20-30 (1996).

[5] Mouralova, K., Kovar, J., Klakurkova, L., Bednar, J., Zahradnicek, R.: Analysis of the surface morphology and topolography of pure aluminium machined using WEDM. Measurement 114, pp. 169-176 (2018) DOI 10.1016/j.measurement.2017.09.040

[6] Mouralova, K., Matousek, R., Kovar, J., Mach, J., Klakurova, L., Bednar, J.: Analyzing the surface layer after WEDM depending on the parameters of a machine for the 16MnCr5 steel Measurement 94, pp. 771-779, (2016) DOI 10.1016/j.measurement.2016.09.028

[7] Petran, M., Hadravsky, M., Egger, M.D., Galambos, R.: Tandem-Scanning Reflected-Light Microscope. Journal of the Optical Sociaty of America 58(5), pp. 661-664 (1968) DOI 10.1364/JOSA.58.000661

[8] Claudette M. St. Croix, C.M., Shand,S.H., Watkins, S.C.:Confocal microscopy: comparisons, applications, and problems. BioTechniques 39(S2-S5), pp. (2005) DOI 10.2144/000112089

[9] Nussbaumer, H.J.: Fast Fourier Transform and Convolution Algorithm, vol. 2, second edn. Spriger-Verlag, Berlin Heidelberg (1982) 